\title{
Terapia Ocupacional para personas con enfermedad crónica en fase avanzada: una visión desde la logoterapia
}

Magda Díaz", Sonia Pachón"*, Leyla Sanabria**", Alicia Trujillo***

En el principio era el sentido y el sentido era la acción.

Victor Frankl

\section{RESUMEN}

Esta investigación exploratoria se relaciona con la atención del terapeuta ocupacional a personas que enfrentan una enfermedad crónica en fase avanzada. El estudio se enmarca dentro del enfoque psicoterapéutico denominado logoterapia, creado hacia 1942 por Viktor Emil Frankl, centrado en la búsqueda de sentido y significado de la vida (1). A partir de la logoterapia se extrapolan principios básicos aplicables a la práctica de Terapia Ocupacional, con el propósito de contribuir en la satisfacción de necesidades de esta población hacia una mejor calidad de vida en el final de su existencia.

Se introduce la logoterapia como marco de referencia filosóficoantropológico que puede orientar la práctica del terapeuta ocupacional,

* Terapeuta Ocupacional Universidad Nacional de Colombia.

* Terapeuta Ocupacional Universidad Nacional de Colombia. Diplomado en Aplicación de la Logoterapia o Tercera Escuela Vienesa de Psicología.

** Terapeuta Ocupacional Universidad Nacional de Colombia. Diplomado en Aplicaciones de la Lototerapia o Tercera Escuela Vienesa de Psicología.

**** Profesora Titular y Emérita Universidad Nacional de Colombia. Directora trabajo de investigación. 
por cuanto ofrece una visión integral del ser humano a partir de sus dimensiones física, psicológica, sociofamiliar y espiritual o noética, siendo esta última en la que se hace mayor énfasis. El producto de la investigación es un conjunto de lineamientos teóricos, sintetizados en diez postulados básicos para el diseño operacional de programas de Terapia Ocupacional en la atención a personas con enfermedad crónica en fase avanzada y su familia.

\section{INTRODUCCIÓN}

El dolor, el sufrimiento y la muerte son experiencias inherentes a la existencia del ser humano, que al ser afrontadas a partir de acciones responsables pueden convertirse en un desafío y una oportunidad de sobreponerse, crecer más allá de sí mismo, cambiar y mejorar. Lo anterior puede estar supeditado por un lado, a los condicionamientos físicos, psicológicos y sociales que la vida misma involucra, y por otro, a la actitud del ser humano para manejar estos condicionamientos y buscar la autotrascendencia.

La enfermedad como proceso natural que puede condicionar la vida del ser humano, generalmente está ligada al sufrimiento pues amenaza la sensación de integridad y entereza de la persona. Con alguna frecuencia quien enfrenta una enfermedad comprende su estado aceptándolo con relativa serenidad; no obstante el impacto que genera el diagnóstico de una enfermedad crónica puede tener connotaciones mucho más profundas para quien lo vive, ya que lo cuestiona acerca de su finitud y de la calidad de sus relaciones, así como sobre el propósito y el significado de su existencia.

Reconocer el proceso que involucra una enfermedad crónica desde las dimensiones física, psicológica, sociofamiliar y espiritual es tarea de los profesionales y de las personas que están relacionadas directamente con esta experiencia (2). Este reconocimiento permite ofrecer a la persona los cuidados necesarios para que viva sus últimos días en forma digna.

En la actualidad, la sociedad industrial ha creado en la persona diversas necesidades que se pueden suplir a partir de los avances tecnológicos que ofrece (3). Tal es el caso de la enfermedad crónica, para la cual la ciencia, en su afán de controlar todo aquello que interfiera en el mantenimiento del bienestar humano, ha tratado 
de suprimir de la conciencia los conceptos de dolor, enfermedad y muerte. Sin embargo, esto mismo ha suscitado un sentimiento de vacío existencial, pues la necesidad más humana que es la de hallar un sentido para la propia vida no se puede satisfacer desde la perspectiva tecnológica. Es entonces cuando el ser humano puede recurrir a su condición espiritual, con el fin de reevaluar su conducta y orientarla hacia la aspiración humana por una existencia significativa (4).

Durante los últimos años se ha visto un aumento en la población mundial con enfermedades crónicas, lo cual ha generado un especial interés de diferentes profesionales en la atención a estas personas, particularmente en la etapa final de su enfermedad.

Terapia Ocupacional es una de las profesiones que se ha involucrado en este campo, teniendo como eje de estudio el desempeño ocupacional, expresado en las actividades que la persona realiza a lo largo de su ciclo vital, especialmente cuando éstas se alteran por problemas físicos, sociales, emocionales o espirituales. El objetivo de este estudio fue proponer lineamientos teóricos que guíen la praxis del terapeuta ocupacional y de esta manera aportar elementos para poder contribuir al mejoramiento de la calidad de vida de las personas con enfermedad crónica en fase avanzada y su familia.

\section{ESTRATEGIA METODOLÓGICA}

Esta investigación exploratoria buscó dar una visión aproximativa en relación con la atención del terapeuta ocupacional a personas con enfermedad crónica en fase avanzada, desde la posición conceptual de la logoterapia. Dado que el tema ha sido poco estudiado y reconocido en la profesión, se hace difícil aún formular hipótesis precisas o de cierta generalidad. Las fuentes para la recopilación de la información se enmarcaron fundamentalmente en la literatura española e inglesa, conocida a través de publicaciones seriadas, libros, consulta a expertos, referencias personales, visita a centros asistenciales, periódicos e Internet.

La revisión de la literatura se cumplió en dos fases. Inicialmente se estudiaron los fundamentos antropológicos de la logoterapia y se identificaron las necesidades de las personas con enfermedad crónica en fase avanzada y sus familias. Posteriormente, a partir de las bases filosóficas de la profesión y de los principios antropológicos 
de la logoterapia se determinaron los lineamientos que pueden orientar la intervención del terapeuta ocupacional con esta población.

\section{RESULTADOS}

En la propuesta "Terapia Ocupacional para personas con enfermedad crónica en fase avanzada: una visión desde la Logoterapia", se determinó que para comprender la realidad de la persona que enfrenta la enfermedad, el terapeuta ocupacional puede recurrir a los principios de la logoterapia. Estos son: la vida tiene sentido en todas las circunstancias; el ser humano es dueño de una voluntad de sentido, es decir, una motivación intrínseca que lo impulsa a actuar para buscar significado en su vida, sintiéndose frustrado o vacío cuando deja de ejercerla; y la persona es libre, dentro de sus limitaciones físicas psicológicas y sociales para consumar el sentido de su existencia (5).

Este enfoque es aplicable a personas que atraviesan una crisis existencial, traducida en una pérdida del sentido de la vida como sucede ante el diagnóstico de una enfermedad crónica. Según la logoterapia, la ocupación es uno de los medios a través del cual se puede alcanzar la realización personal (6), por lo que este enfoque es apropiado para terapia ocupacional.

\section{ORIENTACIÓN DEL TERAPEUTA OCUPACIONAL FRENTE A LAS NECESIDADES DE LA PERSONA CON ENFERMEDAD CRÓNICA EN FASE AVANZADA}

Aunque la enfermedad genera múltiples cambios físicos, psicológicos, sociales y espirituales en el ser humano, en la investigación se hizo énfasis en los síntomas y signos comunes de las enfermedades crónicas que pueden afectar directa o indirectamente el desempeño ocupacional de la persona, perspectiva en la que el terapeuta ocupacional centra su ejercicio profesional.

En el área física el terapeuta ocupacional dirige su atención fundamentalmente hacia las dificultades en las actividades de la vida diaria que involucran el autocuidado o tareas del cuidado personal, la alimentación, el vestido, el baño, la eliminación y la movilidad (7). La ejecución de actividades adquiere valor para la 
persona con enfermedad crónica en fase avanzada al promover el uso de sus habilidades contribuyendo a su bienestar y a satisfacer la necesidad de relacionarse, ser aceptado, proveer seguridad personal y dar sentido a su vida. El valor agregado que tiene la labor del terapeuta ocupacional radica en que fomenta la autonomía de la persona en el desempeño físico, y orienta tanto a la persona como a la familia, en actividades que puede desarrollar en forma independiente, técnicas de organización y ahorro de energía, reorganización de roles y uso de dispositivos de autoayuda.

A nivel psicológico, desde el momento del diagnóstico la persona puede experimentar una serie de trastornos emocionales generados por los cambios en el desempeño a que se va a ver enfrentada, como a la pérdida progresiva de roles y hábitos, de la autonomía en las actividades cotidianas y el aumento del tiempo sin ocupación. La labor del terapeuta ocupacional debe estar dirigida a buscar alternativas que permitan promover, conservar o incrementar la motivación intrínseca de la persona, su autoestima y la capacidad de adaptación y control de su situación durante el transcurso de la enfermedad.

En el plano sociofamiliar, se puede producir una alteración de los roles cotidianos, la comunicación y la dinámica del grupo, lo que conlleva a un proceso de desgaste físico, fragilidad emocional, ansiedad y tendencia a la introversión, ocasionado por los múltiples esfuerzos invertidos en el cuidado de la persona enferma (8). El terapeuta ocupacional debe orientar su intervención con la familia hacia la satisfacción de las necesidades respecto a la reorganización de roles, hábitos y comportamientos, la distribución de tareas y educación para el cuidado de la persona enferma y la autoprotección cuando se generen riesgos para el desempeño ocupacional de los cuidadores, así como realizar intervenciones orientadas a enriquecer el uso de tiempo libre en personas en duelo.

Las necesidades espirituales de las personas con enfermedad crónica están relacionadas con la búsqueda de significado y propósito en la vida, los cuestionamientos acerca del dolor, el sufrimiento, la muerte y la afirmación de valores de creación, experiencia y actitud (9). Los cuestionamientos existenciales de la persona que enfrenta la cercanía de su muerte son el eje de su bienestar y merecen todo el interés del terapeuta ocupacional así como de los demás profesionales que puedan ayudarle. La realización de actividades de la vida diaria, productivas o de esparcimiento que sean significativas permitirán que la persona sienta que su vida aun 
tiene sentido y desarrolle valores de actitud, es decir aquellos que se manifiestan en la persona cuando se encuentra ante situaciones dolorosas e inevitables, de tal forma que le ayuden a disfrutar el día a día a pesar de su condición y aprenda a entender su situación como un hecho natural.

Como resultado de la investigación, se presentan unos lineamientos teóricos de Terapia Ocupacional para la atención a personas con enfermedad crónica en fase avanzada (12), fundamentados en el enfoque psicoterapéutico de la logoterapia. Los lineamientos teóricos pueden sintetizarse en diez postulados que deberían ser la base para el diseño de programas de atención para personas con enfermedades crónicas y sus familias. Estos postulados constituyen el primer paso hacia la posterior elaboración de investigaciones orientadas a la satisfacción de las necesidades de esta población, en busca de una mejor calidad de vida:

1. El ser humano posee una voluntad de sentido o motivación intrínseca que le permite buscar una opción de vida aún en situaciones dolorosas e inevitables como una enfermedad crónica.

2. El sentido de la vida es descubierto a través de significados existenciales llamados valores, cuyo propósito es alcanzar la autotrascendencia en lo que la persona hace, en quienes ama y en situaciones adversas que pueda enfrentar. Los valores se enmarcan en tres categorías: valores de creación, es decir las actividades que el ser humano realiza cotidianamente; valores de experiencia, que subrayan todo aquello que se recibe del mundo como el arte o el amor; $y$ valores de actitud, es decir la forma en que la persona responde ante situaciones dolorosas e inevitables, siendo éstos últimos los que permiten al ser humano alcanzar el grado máximo de significado para la vida en cualquier circunstancia.

3. En la persona con enfermedad crónica en fase avanzada, la ocupación, como parte de los valores de creación, favorece el desarrollo de valores de actitud, especialmente en la última etapa de la vida cuando la cercanía de la muerte se hace inminente y en la que con frecuencia no es posible realizar las actividades cotidianas. El sentido de la vida es percibido cuando el ser humano realiza una acción, experimenta una vivencia o sufre; la ocupación puede concebirse entonces como un medio para enriquecer el mundo interior de las personas, en la medida que ésta le permita dejar huella a pesar de su transitoriedad, 
desarrollar sus habilidades, cumplir con sus expectativas o mantener su independencia.

4. Una enfermedad crónica genera cambios físicos, psicológicos, sociofamiliares y espirituales en la persona que la enfrenta, ocasionando la pérdida progresiva de roles, hábitos y autonomía en el desempeño de las actividades de la vida diaria, trabajo y tiempo libre, es decir en su desempeño ocupacional.

5. Los objetivos de terapia ocupacional en la atención a personas con enfermedad crónica en fase avanzada deben orientarse primordialmente a la búsqueda del sentido y el significado de la vida. El proceso terapéutico, guiado por el terapeuta ocupacional a través de la actividad con propósito, la relación que se establece con la persona y su familia y el ambiente terapéutico, pretende promover en quien enfrenta la enfermedad un grado de crecimiento nunca antes logrado, con una madurez plena, profunda, auténtica y con un verdadero valor personal.

6. Los medios de atención utilizados por el terapeuta ocupacional, tienen como fin mejorar la calidad de vida de quienes enfrentan una enfermedad crónica en fase avanzada, favoreciendo su estado emocional y motivándolos en la búsqueda de algo más allá de sí mismos, dándole un carácter singular a la forma en que afrontan este proceso.

7. El establecimiento de una relación terapéutica que permita a la persona plasmar en vivencias los significados en forma más plena y a que progrese en esa vivencia, permitirá que ella entienda y se adapte mejor a su situación, manteniendo un elevado nivel de autoconfianza y participando en la toma de decisiones sobre el proceso terapéutico que se realiza.

8. Durante el proceso terapéutico, la actividad cumple la función de ofrecer un signo a través del cual el terapeuta puede descubrir y comprender la forma en que la enfermedad está afectando a la persona. El resultado de la actividad y su proceso de ejecución pueden reflejar el interior de la persona y bajo la orientación del terapeuta ocupacional posibilitar una modificación actitudinal de manera que la persona pueda transformar su experiencia personal en una oportunidad para madurar interiormente, dando sentido a la situación que vive. 
9. En terapia ocupacional, el ambiente y el significado particular que éste adquiere cumplen una función fundamental más allá de las dimensiones física, psicológica, social y espiritual de la persona a lo largo de su ciclo vital. Para una persona que enfrenta una enfermedad crónica, el ambiente puede adquirir un nuevo significado en la medida en que le proporcione bienestar y seguridad al representar sus deseos y esto le motive la búsqueda de sentido para su vida.

10. La dimensión noética o espiritual de la persona con enfermedad crónica en fase avanzada cobra especial importancia cuando por el proceso de enfermedad se afecta la adaptación a su ambiente con el consecuente impacto negativo en la ocupación. Es a través de la espiritualidad que la persona puede trascender los problemas de orden físico, psicológico y sociofamiliar asociados a la enfermedad (13).

\section{CONCLUSIONES}

Los seres humanos atraviesan a lo largo de su ciclo vital una serie de circunstancias que pueden involucrar una amenaza a su integridad. Tal es el caso de una enfermedad crónica, la cual pone de manifiesto la interrelación entre las dimensiones del ser humano. Es así como un problemà de orden específicamente biológico es capaz de generar una serie de reacciones psicológicas, sociales y espirituales que fundamentalmente se traducen en cambios en el desempeño ocupacional de la persona.

El deterioro progresivo al que se enfrenta la persona con enfermedad crónica en fase avanzada en las dimensiones física, psicológica, sociofamiliar y espiritual implica un especial planteamiento de los objetivos terapéuticos (10). Ante tọdo, el terapeuta ocupacional debe dirigir su intervención en la búsqueda de sentido y significado a la vida de la persona a través del establecimiento de la relación terapéutica, el uso de actividades con significado y la adecuación de un ambiente terapéutico, orientados a la satisfacción de las necesidades de la persona y su familia.

Profesionales de diferentes disciplinas, especialistas en tanatología o estudio de la muerte, afirman que la ocupación es un elemento fundamental para hallar significado en medio de circunstancias adversas (11) y aconsejan genuinamente que 
quienes enfrentan situaciones críticas, como la cercanía de la muerte, desarrollen actividades que sean de su agrado, de tal forma que puedan construir un nuevo y último significado para su vida. De esta afirmación se deriva que el terapeuta ocupacional, por poseer el conocimiento científico y las herramientas necesarias para contribuir al mejoramiento de la calidad de vida a través de actividades con propósito, está en capacidad de optimizar el desempeño ocupacional de quienes enfrentan enfermedades crónicas.

\section{LISTA DE REFERENCIAS}

1. Bazzi, Tullio. Guía de la logoterapia: Humanización de la psicoterapia. Barcelona: Editorial Herder. 1989.

2. Astudillo, Wilson y Mendinueta, Carmen. Cuidados del enfermo en fase terminal y atención a su familia. Pamplona: Ediciones Universidad de Navarra S.A. Tercera edición. 1997.

3. Frankl, Viktor E. En el principio era el sentido: reflexiones en torno al ser humano. Buenos Aires: Ediciones Paidós Ibérica S.A., 2000.

4. Acevedo, Gerónimo. La búsqueda de sentido y su efecto terapéutico: desde la perspectiva de la logoterapia de Viktor Frankl. Buenos Aires: Ediciones Fundación Argentina de Logoterapia "Viktor Frankl". 1985.

5. Fabry, Joseph B. La búsqueda de significado: la logoterapia aplicada a la vida. México: Fondo de Cultura Económica. 1977.

6. Guttmann, David. Logoterapia para profesionales: trabajo social significativo. Bilbao: Ediciones Desclee de Brouwer: 1998.

7. Hopkins, Helen y Smith, Helen. Terapia Ocupacional. Octava edición, España: Editorial Médica Panamericana. 1998.

8. Brusco, Angelo. Humanización de la asistencia al enfermo. Santander: Editorial Sal Terrae, Centro de Humanización de la Salud. 1999.

9. De Lima, Liliana y Rincón, Hernán. "La evaluación emocional en los pacientes terminales". En: Revista Enlace cuadernos de psiquiatría. Número 2. Junio, 1999. 


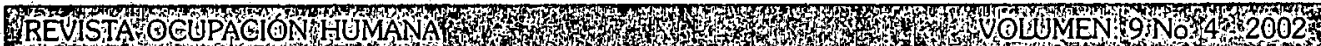

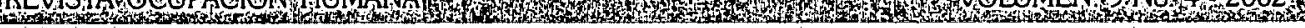

10. .. Durante Molina, Pilar. Terapia Ocupacional en salud mental: principios y práctica. Barcelonia: Masson S.A. 1998.

11. Fonnegra de Jaramillo, Isa. De carà a la muerte: cómo afrontar lás penas, el dolor y la muerte para vivir más plenamente. Santafé de Bógotá: Intermedio Editorès. 1999.

12. Díaz, M.; Pachón, J.; Sanabria, L. Terapia Ocupacional para personas con enfermedad crónica en fase avanzada: una visión desde la logoterapia. Tesis de grado. Facultad de Medicina. Universidad Nacional de Colombia. Bogotá, 2001.

13. Peloquin, Suzanne. "The spiritual Depth of Occupation: Making Worlds and Making lives". In: American Journal of Occupational Therapy. Vol. 51 No. 3, marzo de 1997.

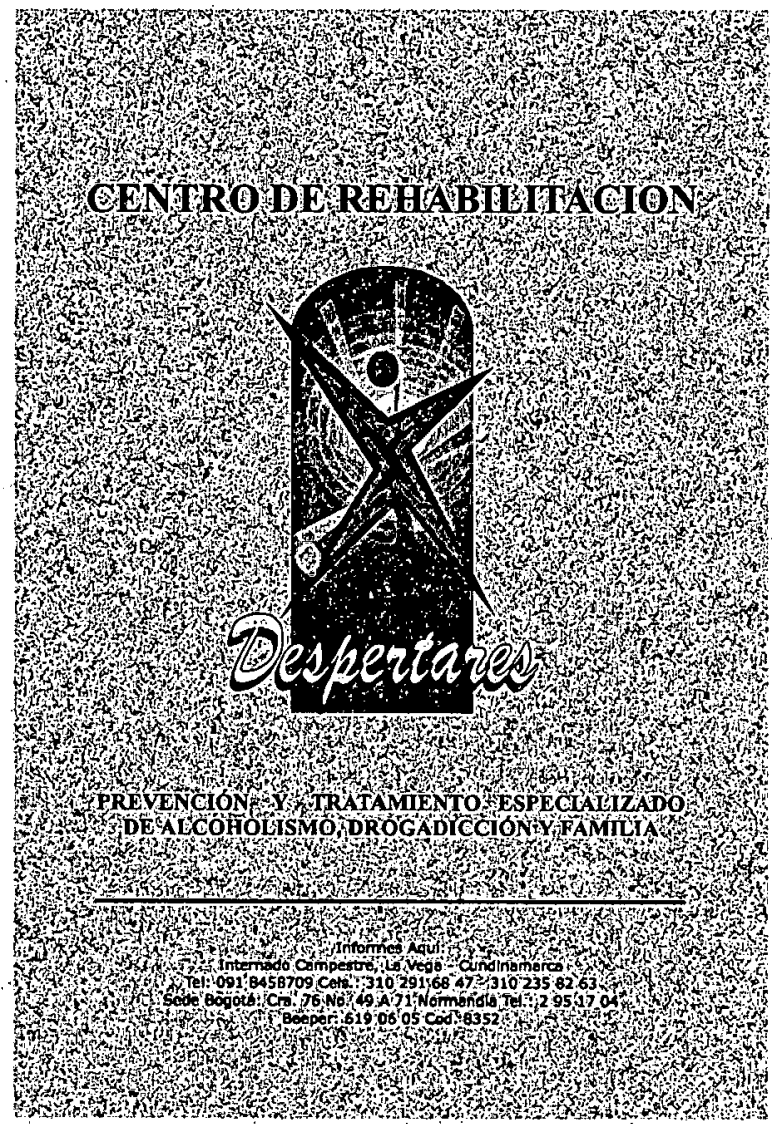

